\title{
DAS PROBLEMATISCHE VERHÄLTNIS ZWISCHEN LEBENSWELT UND TRANSZENDENTALER KONSTITUTION
}

\author{
DOMINIQUE PRADELLE
}

\begin{abstract}
The problematic relation between the life-world and transcendental constitution

This paper focuses on the possibility of transcendental constitution of the life-world: is it possible to constitute the life-world, and does the concept of life-world modify something essential in the concept of transcendental constitution? There were in fact interpretations of Husserlian thought which admitted that in the last period Husserl gave up the method of transcendental constitution and defended the thesis that the world as totality is unconstituable. In this paper we try to demonstrate that Husserl never gave up transcendental constitution, and to clarify structures and specific problems of the constitution of the life-world. Moreover we point to a methodological difficulty: the constitution of the world is identical with the universal and stratified constitution of the strates of objectivity, and is in accordance with the law of foundation of the strates; but there is a fundamental contradiction between that stratified constitution in naturalistic attitude and the real experience of the world in personalistic attitude.
\end{abstract}

Die wesentliche Frage, die wir in diesem Text behandeln werden, ist folgende: Ist die Lebenswelt im Sinne der transzendentalen Konstitution konstituierbar oder nicht? Modifiziert ferner der Begriff der Lebenswelt etwas Zentrales und Wesentliches am Begriff der transzendentalen Konstitution oder nicht? Zwingt uns die Ausarbeitung dieses Begriffs dazu, auf den theoretischen Entwurf der transzendentalen Konstitution zu verzichten, oder lediglich dazu, dessen besonderen idealistischen Begriff loszulassen? Creative Commons Attribution License (http://creativecommons.org/licenses/by/4.0). 


\section{Hat Husserl den Entwurf der transzendentalen Konstitution der gegenstände Wegen der Idee der Lebenswelt fallengelassen?}

Unseren Ausgangspunkt nehmen wir bei einem Text von Paul Ricœur, der im Jahre 1980 erschienen ist und dessen Titel „Loriginaire et la question-en-retour dans la Krisis“ [„Das Ursprüngliche und die Rückfrage in Husserls Krisis“] lautet. ${ }^{1}$ In diesem Text hebt Ricœur eine besondere Schwierigkeit hervor, die in Husserls Bestimmung der Lebenswelt liegt: Einerseits wird die Lebenswelt als der unbefragte und letzte Grund aller Voraussetzung herausgestellt; andererseits wiederholt aber Husserl den Cartesianischen Zweifel, der nicht nur die Geltung der wissenschaftlichen Lehren umspannt, sondern auch die der Welt des vorwissenschaftlichen Lebens. Gibt es also so etwas wie einen Widerspruch in Husserls Denken oder mindestens eine theoretische Spannung zwischen diesen beiden entgegengesetzten Orientierungen?

Tatsächlich - so lautet Ricœurs Antwort - gibt es hier keine Unverträglichkeit beider Thesen unter der Bedingung, dass man folgende scharfe Unterscheidung im Sinn behält: diejenige zwischen der „ontologischen Funktion der Lebenswelt als einer vor aller Deutung daseienden Welt“2 und deren "erkenntnistheoretischer Funktion, als einer auf Geltung Anspruch erhebenden Welt“3. Ihrer erkenntnistheoretischen Funktion nach ist die Lebenswelt zugleich gründender Boden für alle wissenschaftlichen Konstruktionen und Ausweisungsprinzip für diese Konstruktionen; ${ }^{4}$ aber deren ontologische Funktion ist insofern von viel wichtigerer Bedeutung, als der Lebensweltbegriff zugleich die negative und polemische Funktion besitzt, „sich einem entgegengesetzten Anspruch des Bewusstseins zu widersetzen, als Ursprung und Meister alles Sinnes zu gelten. Die erste Funktion des Lebensweltbegriffs besteht genau darin, diesen Anspruch zunichte zu machen“ ${ }^{5}$. Nach Ricœur gibt es also einen Gegensatz zwischen der epistemologischen und der ontologischen Funktion der Lebenswelt, d. h. zwischen dem „erkenntnistheoretischen Primat der Wissenschaftsidee“ und der „ontologischen Vorgängigkeit der

Ricoeur Paul, „L'originaire et la question-en-retour dans la Krisis de Husserl“, in Textes pour Emmanuel Levinas, hrsg. von François Laruelle, Paris, Jean-Michel Place éditeur, 1980, S. 171-174, wiederabgedruckt in Ricoeur Paul, À lécole de la phénoménologie, Paris, Vrin, 1987, S. 289-292.

2 Ibid., S. 173: „la fonction ontologique de la Lebenswelt, comme étant là avant toute interprétation“ (À lécole..., S. 291).

3 Ebenda: „sa fonction épistémologique en tant que prétendant à la validité“.

Ibid., S. 176 (À lécole..., S. 294).

5 Ebenda: „[...] s'oppose à une prétention adverse, celle de la conscience à sériger en origine et maitre du sens. La première fonction du concept de Lebenswelt est de ruiner cette prétention“. 
Lebenswelt “6. Einerseits fordert nämlich die Wissenschaftsidee, dass das Bewusstsein als letzter und absoluter Grund alles Sinnes und alles Seins gelte; andererseits „reduziert die Rückkehr zur Lebenswelt diese Hybris durch die Behauptung, dass ihr die Lebenswelt immer schon vorangegangen ist "7. Nach Ricœurs Meinung gibt es also in Husserls spätem Denken eine Wendung zu einem radikalen Realismus der Lebenswelt, der diese als vom Bewusstsein unkonstituierbar und für alles Subjekt absolut vorgegeben anerkennt.

Ist aber Ricœurs Interpretation eine richtige Auslegung von Husserls spätem Denken? Hat Husserl wirklich darauf verzichtet, die transzendentale Konstitution alles mundanen Seienden und der Welt selbst aufzuklären?

Für uns erscheint Ricœurs Meinung als eine falsche Interpretation, die eine irreführende Verwechslung zwischen der gründenden Funktion der Lebenswelt den Wissenschaften gegenüber und der angeblich absoluten ontologischen Vorgegebenheit der Lebenswelt dem Bewusstsein gegenüber vollzieht. Das hatte schon Walter Biemel im Jahre 1959 in seinem Vortrag „Die Philosophie als strenge Wissenschaft“ hervorgehoben. ${ }^{8}$ Man behauptet oft, schreibt W. Biemel, dass das Entscheidendste in Husserls Krisis in der Rückkehr von der wahren Welt der Wissenschaften her bis zur vorwissenschaftlichen Lebenswelt läge, als ob diese Gegenüberstellung den philosophischen Endzweck dieses letzten Buchs lieferte; im Gegenteil gilt die Rückkehr zur Lebenswelt nur insofern, als damit die Frage der transzendentalen Konstitution der wissenschaftlichen Welt nachher gestellt werden kann; ${ }^{9}$ dieser erste methodische Schritt gilt also absolut nicht als letzter Punkt, sondern nur als Vorstufe der Aufgabe der transzendentalen Konstitution: „Im Gegenteil soll die Erforschung der Lebenswelt das konstituierende Leisten des transzendentalen Egos ans Licht bringen. Die Lebenswelt (die alltägliche Welt) ist keine letzte Stufe, sondern wird selbst konstituiert" ${ }^{\prime 10}$.

6 Ibid., S. 177: „le primat épistémologique de l'idée de science“ und „l'antériorité ontologique du monde de la vie" (À l'école..., S. 295).

7 Ibid., S. 176: „Le retour au monde de la vie réduit cette hybris en assertant que le monde l'a dès toujours précédée“ (À lécole..., S. 294).

8 Biemel Walter, „La philosophie comme science rigoureuse“ und Diskussion von K. Kuypers Vortrag „La conception de la philosophie comme science rigoureuse et les fondements des sciences chez Husserl“ [„Die Auffassung der Philosophie als strenger Wissenschaft und die Fundamente der Wissenschaften"], in Husserl, Cahiers de Royaumont, Philosophie n III, Paris, Minuit, 1959, S. 58 und S. 85.

9 Ibid., S. 58.

10 Ibid., Diskussion, S. 85: „M. Kuypers oppose la recherche de la Lebenswelt à celle de l'ego transcendantal, comme si par la recherche de la Lebenswelt létude de l'ego transcendental pourrait être remplacée. Mais ce nest nullement le cas. Au contraire, la recherche de la Lebenswelt doit mettre en lumière l'activité constituante de l'ego transcendantal. La Lebenswelt (le monde quotidien) n'est pas une dernière couche, mais elle est elle-même constituée, et la mise au jour de cette constitution doit 
Diese wesentliche These von W. Biemel möchten wir zuerst ausweisen; dazu werden wir zwischen der erkenntnistheoretischen und der ontologischen Funktionen der Lebenswelt streng unterscheiden müssen.

\section{Die doppelte Funktion der Lebenswelt: vorausgesetzter Grund aller wissenschaftlichen Erkenntnis und erstes Thema der transzendentalen Konstitution}

Mehrfaches Verhältnis zwischen Lebenswelt und wissenschaftlicher Welt, vorwissenschaftlicher Anschauung und objektiv-wissenschaftlichem Denken

Erstens gilt das Problem der Lebenswelt „als ein Teilproblem im allgemeinen Problem der objektiven Wissenschaft "11. „So ist das Problem der Lebenswelt [...] nur ein partielles Thema innerhalb des bezeichneten Ganzen der objektiven Wissenschaft (nämlich im Dienst ihrer vollen Begründung)“12. D. h. im Rahmen der Aufgabe der transzendentalen Konstitution hat die Lebenswelt eine begründende Funktion, insofern als sie „ständiger Boden“13 für alle wissenschaftlichen Konstruktionen und Ausweisungen ist; in dieser erkenntnistheoretischen Hinsicht lässt sich das Primat der anschaulichen Lebenswelt als Unterlage für alle darauf sich aufbauenden Gegenstände des wissenschaftlichen Denkens herleiten: „Das wirklich Erste ist die ,bloss subjektiv-relative' Anschauung des vorwissenschaftlichen Weltlebens"14. Die Frage der Lebenswelt erweist sich also als die „Frage nach dem Verhältnis von objektiv-wissenschaftlichem Denken und Anschauung "15: und zwischen der wissenschaftlichen Welt und der Lebenswelt gibt es ein mehrfaches Verhältnis.

Erstens ein Einbezogenheitsverhältnis: Die wissenschaftlichen Praxeis und Lebensinteressen erweisen sich nämlich als besondere und teilhafte Formen innerhalb der Allheit der möglichen Praxeis und Lebensinteressen; die theoretische Pra-

nous révéler das anonyme Leisten (donc l'activité anonyme) de l'ego transcendantal. “ C. Romano hat in einem relativ neu erschienenen Buch diese Deutung des späten Husserlschen Denkens von Kuypers und Ricœur wiederholt, die wir ebenso wie W. Biemel für falsch halten; vgl. Romano Claude, Au cœur de la raison, la phénoménologie, Paris, Gallimard, 2010, S. 928.

11 Husserl Edmund, Die Krisis der europäischen Wissenschaften und die transzendentale Phänomenologie (= Krisis), §33, Husserliana Bd. VI (= Hua VI), Den Haag, M. Nijhoff Publishers, 1976, S. 123.

12 Ibid., \$33, Hua VI, S. 125.

13 Ibid., \$33, Hua VI, S. 124.

14 Ibid., \$ 34, Hua VI, S. 127.

15 Ibid., \$ 34f, Hua VI, S. 137. 
xis, die für die Wissenschaften charakteristisch ist, erweist sich also nur als Teil der möglichen menschlichen oder subjektiven Leistungen. Und die eine und einheitliche Lebenswelt bildet das vorgegebene Gebiet, innerhalb dessen sich alle diese subjektiven Aktivitäten entfalten können: „Es ist natürlich die eine, allgemeinsame Erfahrungswelt, in der auch Einstein und jeder Forscher sich als Mensch, und auch während all seines forschenden Tuns, weiß" 16 . In dieser Hinsicht bestimmt sich der Lebensweltbegriff als umgreifender Begriff, d. h. als Allbegriff, der alle möglichen Formen der subjektiven Leistung einschließt.

Zweitens gibt es zwischen der wissenschaftlichen Welt und der Lebenswelt ein Gegenüberstellungs- und Idealisierungsverhältnis: Es liegt tatsächlich im Sinne der Aufgabe der Wissenschaften, objektive Gültigkeit zu gewinnen (d. h. Geltung für jedermann und für alle Zeiten, intersubjektive und allzeitliche Gültigkeit), was aber voraussetzt, die subjektive Relativität der sinnlichen Anschauungen und der Situationswahrheiten zu überwinden: „Der Konstrast dazu [d. h. zum Stempel bloss ,subjektiv-relativ'] bestimmt, wie wir sagten, den Sinn der ,objektiven 'Aufgabenstellung. Dieses ,Subjektiv-Relative` soll ,überwunden 'werden; man kann und soll ihm zuordnen ein hypothetisches An-sich-Sein, ein Substrat für logisch-mathematische ,Wahrheiten an sich"“17. Dazu dienen die Mathematisierung der Anschauungswelt, d. h. insgesamt die Verwerfung aller sinnlichen Fülle oder Eigenschaften (visueller und taktueller Eigenschaften: Farben usw.); die Exaktmachung oder Idealisierung der anexakt-morphologischen Begriffe (gerade, eben, rund, eisförmig usw.), die diese anexakten Gebilde zu exakten geometrischen Gebilden macht (Gerade, Ebene, Zirkel, Ellipse usw.); die ideale Vervollkommnung der realen technischen Praxis und deren Umstellung in „eine ideale Praxis eines reinen Denkens" ${ }^{18}$; und schließlich die Mitmathematisierung oder indirekte Mathematisierung der sinnlichen Qualitäten, die sie als geregelt verschwisterte Korrelate von physikalisch-mathematischen Gestalten behandelt. ${ }^{19}$

Drittens gibt es zwischen den beiden Weltformen ein Begründungsverhältnis: In der Tat erscheint die subjektiv-relative Lebenswelt als „Evidenzquelle, Bewährungsquelle“, „als das [...] die theoretisch-logische Seinsgeltung letztlich Begründende ${ }^{\prime 20}$-, und dies insofern, als die Maßstäbe, Teilstriche und Messungsergebnisse direkt anschaulich und als wirklich seiend wahrgenommen werden müssen.

\footnotetext{
Ibid., $\$ 34$ b, Hua VI, S. 128.

Ibid., \$34b, Hua VI, S. 129.

Ibid., $\$ 9 \mathrm{a}, \mathrm{Hua}$ VI, S. $22-25$.

Ibid., $\$ 9$ b-c, Hua VI, S. 28, 31-36.

Ibid., \$34b, Hua VI, S. 129.
} 
Viertens und letztens gibt es zwischen beiden Weltformen ein Unterschiebungs- und Verlängerungsvershältnis: Schon im vorwissenschaftlichen Leben vollzieht sich eine kunstlose und anexakte Induktion oder Voraussicht der anschaulichen Phänomene, insofern als die Dinge der anschaulichen Welt „sozusagen ihre ,Gewohnheiten ' haben, sich unter typisch ähnlichen Umständen ähnlich zu verhalten “21 oder einem geregelten Gesamtstil zu folgen; im wissenschaftlichen Denken vollzieht sich und verlängert sich derselbe induktive Prozess, aber in der Form einer exaktgemachten, mathematischen und methodischen Induktion: Durch das physikalische Denken leisten wir also „eine ins Unendliche erweiterte Voraussicht ${ }^{\text {"22 }}$, die nur eine verlängerte, idealisierte und kunstvolle Form der wahrnehmungsmäßigen Vormeinung ist und die die idealisierte mathematisch-physikalische Natur der vorwissenschaftlich anschaulichen Natur unterschiebt. ${ }^{23}$

\section{Doppelte Bestimmung des Lebensweltbegriffs}

Welche Bestimmtheiten der Lebenswelt ergeben sich daraus?

Daraus folgt eine doppelte Bestimmung der Lebenswelt, die von Husserl im $\$ 34$ der Krisis ganz klar deutlich gemacht und hervorgehoben wird: „Konkrete Lebenswelt also zugleich für die ,wissenschaftlich wahre Welt der gründende Boden und zugleich in ihrer eigenen universalen Konkretion sie befassend - wie ist das zu verstehen $[\ldots]$ ? “24.

Einerseits ergibt sich die Lebenswelt als die rein anschauliche, wahrnehmungsmäßige und erfahrungsmäßige Sinnenwelt, die der idealisierten und substruierten wissenschaftlichen Welt entgegengesetzt ist: „Die Lebenswelt ist ein Reich ursprünglicher Evidenzen“25, d. h. mit dem Gebiete identisch, worin die Selbstgebung individueller Gegenstände stattfinden kann; und diese Selbstgebung ist primär wahrnehmungsmäßige oder sinnliche Auffassung des Gegenstands, die diesen als mit sinnlichen Qualitäten behaftet gibt. Aber diese reine Sinnenwelt wird auch von allen besonderen kulturellen Welten und Interessenhorizonten streng unterschieden: ${ }^{26}$ für das alltägliche Leben wird allein die besondere Interessenwelt als Gebiet für Lebensinteressen und Lebenszwecke thematisch, während dagegen die Lebenswelt als sinnlicher und allen Interessen vorangehender Grund unthematisch

\footnotetext{
Ibid., \$9b, Hua VI, S. 28.

Ibid., $\$ 9$ h, Hua VI, S. 51, und $\$ 34$ d, Hua VI, S. 130, Fussnote.

Ibid., \$9h, Hua VI, S. 50.

Ibid., \$34e, Hua VI, S. 134.

Ibid., \$34d, Hua VI, S. 130.

Ibid., Beilage XVII, Hua VI, S. 458.
} 
bleibt. ${ }^{27}$ Diese lebensweltliche Unterlage für alle kulturellen Praxeis gibt den minimalistischen Lebensweltbegriff, also denjenigen eines rein sinnlichen und wahrnehmbaren Fundaments für alle kulturellen Aktivitäten und wissenschaftlichen Konstruktionen; nur in dieser Hinsicht darf Husserl für gültig halten, dass die Lebenswelt „bleibt, als die sie ist, in ihrer eigenen Wesensstruktur, in ihrem eigenen konkreten Kausalstil ungeändert, was immer wir kunstlos oder mit Kunst tun" ${ }^{28}$ d. h. dass sie eine ungeschichtliche und unveränderliche Wesensstruktur besitzt.

Andererseits bestimmt sich aber die Lebenswelt nicht nur als Unterlage für alle praktischen Interessen, sondern auch als intentionales Korrelat dieser Interessen, als konkrete Kulturwelt oder als Umwelt für alle praktischen Interessen - d. h. als eine konkrete Umwelt, die kulturelle und geschichtliche Veränderlichkeit annimmt und sich für die „Neger am Kongo" und für die „chinesischen Bauer“ als verschieden erweist. ${ }^{29}$ Dieser Lebensweltbegriff umgreift alle besonderen Lebensweltformen, die intentionale Korrelate von besonderen kulturellen und geschichtlichen Lebensformen sind, und bildet zugleich einen maximalistischen und besonderen Lebensweltbegriff, der nicht die sinnliche unkulturelle Unterlage für kulturelle Aktivitäten besagt, sondern umgekehrt kulturelle Prädikaten auch annimmt. Nur wenn wir den konkreten Gesamtinbegriff der kulturellen Umwelten betrachten, ergibt sich der zugleich maximalistische und allgemeine Lebensweltbegriff als „das Gebiet der Ziele, des schon Erzielten und Zu-erzielenden in seiner eigenen Universalität,

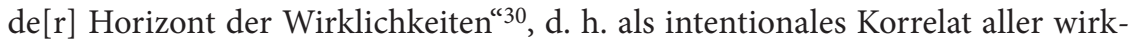
lichen und möglichen praktischen und kulturellen Aktivitäten.

Wir müssen also einen minimalistischen und abstrakten Begriff und einen maximalistischen und konkreten Begriff von Lebenswelt scharf unterscheiden. Einerseits ist diese die vorgegebene, allgemeine und rein sinnliche Welt, die „immer schon da, im voraus für uns seiend, ,Boden' für alle, ob theoretische oder extratheoretische Praxis“31, ist; andererseits ist sie „der Horizont, der alle unsere Ziele, alle unsere Zwecke [...] strömend-ständig befaßt“"32. Erstens wird die Lebenswelt als ungeschichtlicher, unkultureller und rein sinnlicher Boden bestimmt, zweitens dagegen als kultureller und geschichtlicher Horizont mit ihrem ganzen konkreten Gehalt aufgefasst.

\footnotetext{
Ibid., Beilage XVII, Hua VI, S. 461: die Lebenswelt ist „das ,Gebiet', das allen Zwecken [...] vorangeht".

28 Ibid., $\$ 9$ h, Hua VI, S. 51, und $\S 36$, Hua VI, S. 142.

9 Ibid., \$36, Hua VI, S. 142.

Ibid., Beilage XVII, Hua VI, S. 461.

Ibid., \$37, Hua VI, S. 145.

Ibid., $\$ 38$, Hua VI, S. 147.
} 
Die transzendentale Wissenschaftlichkeit als besondere Art der Wissenschaftlichkeit

Es muss drittens eine besondere Art von Wissenschaftlichkeit geben, die der Thematisierung und Behandlung der Lebenwelt zu entsprechen hat: „die richtige Fassung der Lebenswelt und die Methode einer ihr angemessenen ,wissenschaftlichen Behandlung', wo doch ,objektive' Wissenschaftlichkeit außer Frage bleiben soll“33. Welche ist aber diese angemessene methodische Behandlung der Lebenswelt? Wird sie vom Wesen der Lebenswelt bestimmt? Und enthält sie die Aufklärung der transzendentalen Konstitution der umweltlichen Gegenstände oder schliesst sie diese vielmehr aus?

Näher betrachtet soll die wissenschaftliche Behandlung der Lebenswelt drei Verfahrungsweisen einschließen, wodurch eine besondere Spezies von Wissenschaftlichkeit bestimmt wird, die vom Typus der objektiven Wissenschaftlichkeit scharf unterschieden werden muss.

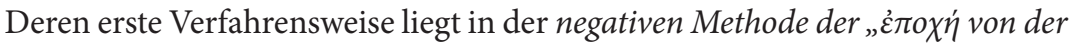
objektiven Wissenschaft" ${ }^{\text {"34 }}$, d. h. des Abbaus aller wissenschaftlichen Idealisierungen oder der „Vollzugsausschaltung “ 35 an allem wissenschaftlichen Interesse für das An-sich-Wahre. Da sie am Ideal der intersubjektiven oder absoluten Gültigkeit orientiert bleibt und auf das intersubjektiv und allzeitlich Wahre abzielt, hat die moderne Physik eine Reduktion aller qualitativen Eigenschaften und eine idealisierende Geometrisierung der wirklichen Körper und wahrnehmungsmäßigen Gestalten vollzogen, um eine an sich gültige und aus exakt geometrischen Figuren bestehende Natur aufzubauen und zu bestimmen. Dagegen soll erstens Wissenschaft der Lebenswelt eine Umkehrung dieses idealisierenden Aufbaus leisten, um zur Welt der wahrgenommenen und sinnlichen Körper zurückzukehren; diese Methode der Rückkehr zum Subjektiv-Relativen (d. h. zu den Sinnesdaten: Farben, haptische Daten, usw.) erlaubt uns, alle transzendentalen Leistungen der idealisierenden Wissenschaften herauszustellen und als solche ans Licht zu bringen.

Deren zweite Verfahrensweise liegt in der positiven Methode der Wesensschau oder Wesensanschauung, welche die Methode einer deskriptiven Ontologie der Lebenswelt bildet: „[...] dass doch die Lebenswelt in ihren allen Relativitäten ihre allgemeine Struktur hat. Diese allgemeine Struktur, an die alles relativ

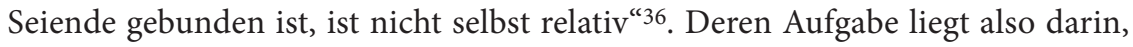

\footnotetext{
Ibid., $\$ 33$, Hua VI, S. 128.

Ibid., $\$ 35$, Hua VI, S. 138.

Ibid., \$35, Hua VI, S. 140.

Ibid., \$36, Hua VI, S. 142.
} 
„das Kategoriale der Lebenswelt“ aufzusuchen, d. h. „die formal-allgemeinsten Strukturen der Lebenswelt" durch eidetische Anschauung herauszustellen, oder „das Formal-Allgemeine, das an der Lebenswelt in allem Wandel der Relativitäten invariant Verbleibende “37, aufzulösen. Jede eidetische Variation der erfahrungsmäßigen Welt lässt in der Tat gewisse wesensnotwendige Strukturformen invariant stehenbleiben, ${ }^{38}$ die als Einheitsformen zu bestimmen sind, denen die reale Gliederung der Welt in Realitätsregionen entspricht: nämlich die Allheitsformen Zeit, Raum, Materie, Leben, Subjektivität, Kulturwelt usw., welche der ontischen Regionen der sinnlichen materialen Dinge, der Lebewesen, der Animalien, der Personen, der Gebrauchsdinge und sonstigen kulturellen Dinge usw. entsprechen. Diese Einheitsformen zu bestimmen bildet die eigene Aufgabe einer Ontologie der Lebenswelt, d. h. einer rein deskriptiven Eidetik der vorwissenschaftlichen Welt. ${ }^{39}$

Bestimmt aber diese Aufgabe die einzige und letzte Wissenschaft der Lebenswelt, so dass deren Beschreibung das Vorbild der höchsten und letzten Rationalität lieferte? Keineswegs! Die Beschreibung der eidetischen gegenständlichen Strukturen der Lebenswelt macht die letztbegründende Wissenschaft dieser Welt absolut nicht aus; außerdem und nachdem gibt es noch die letztbegründende Thematisierung „des universalen Korrelationsapriori von Erfahrungsgegenstand und Gegebenheitsweisen“40 oder der „Korrelation von Aussehen und Aussehendem als solchen " 41 , deren systematische Ausarbeitung die Hauptaufgabe der transzendentalen Phänomenologie ist. Eine wesentliche methodische und philosophische Aufgabe besteht nämlich darin, die „philosophische Naivität“42 zu überwinden, die nach Husserl die zeitgenössische Philosophie charakterisiert: d. h. den Realismus der Lebenswelt, der den untranszendentalen Phänomenologien zugehört (nämlich Heideggers und Schelers). Diesem untranszendentalen Realismus entgegen gilt es, die für uns seiende Welt verständlich zu machen, was bedeutet, sie „verständlich als ein Sinngebilde aus den elementaren Intentionalitäten " 43 zu machen: Die Lebenswelt echt aufzuklären heißt, sie als einen von den Bewusstseinssynthesen konstituierten Sinn verständlich zu machen. Die höchste und letzte Verständlichkeit liegt also nicht in der Bejahung der letzten Selbstgebung der subjektiv-relativen

37 Ibid., $\$ 37$, Hua VI, S. 145.

38 Husserl E., Formale und transzendentale Logik, $\$ 103$, hrsg. v. P. Janssen, Den Haag, M. Nijhoff, 1974, Hua XVII, S. 278.

39 Husserl E., Die Krisis der europäischen Wissenschaften und die transzendentale Phänomenologie, op. cit., $\$ 51$, Hua VI, S. 176.

40 Ibid., $\$ 48$, Hua VI, S. 169, Fussnote.

41 Ibid., $\$ 48$, Hua VI, S. 168.

42 Ibid., \$ 49, Hua VI, S. 170.

43 Ibid., $\$ 49$, Hua VI, S. 171. 
Lebenswelt, sondern in der Aufklärung ihrer transzendentalen Konstitution - und dies der ontologischen Thesis gemäß, wonach alles Seiende überhaupt seine Konstitutionsweise im Leisten der transzendentalen Subjektivität hat.

Letztlich bildet die Lebenswelt keine Ausnahme, die ihren eigenen Ort ganz außer der grundsätzlichen ontologischen Thesis der transzendentalen Phänomenologie hätte, wonach alles Seiende überhaupt ein „innerhalb des Ego sich konstituierender Seinssinn“ oder ein in deren intentionalen Leistung „konstituiertes

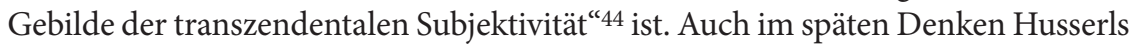
gibt es keine Gegenüberstellung zwischen den einzelnen endlichen Seienden und der Welt als dem All des Seienden: So wie alles Seiende überhaupt als Gebilde der transzendentalen Leistungen der Subjektivität verständlich wird, wird auch die Welt als ein solches Gebilde verständlich. Wieso?

Wie bestimmt sich der Weltbegriff seinem Wesen nach? Faktisch ist zwar die Welt „das All der Dinge“ 45 , d. h. der einzelnen Seienden. Aber diesem faktischen Weltbegriff müssen wir den eidetischen Weltbegriff gegenüberstellen; ihrem Wesen nach bestimmt sich nämlich die Welt als die geordnete Gesamtstruktur aller regionalen Wesen bzw. Strukturformen, die sich dem Fundierungsverhältnis nach aufbauen und sich in eine Stufenfolge einordnen lassen: materielle Dinge, Lebewesen, Animalien, Personen, Gemeinschaften, Kulturobjekte, Idealitäten... Demgemäß muss die Aufgabe der transzendentalen Konstitution methodisch fortschreiten.

Einerseits bestimmt sich konkret Weltevidenz oder Weltkonstitution nach den verschiedenen Evidenzmodi, die den verschiedenen Arten von mundanen Gegenständen entsprechen: „Evidenz ist nicht eine leere Allgemeinheit, sondern differenziert sich nach den Arten, Gattungen, regionalen Kategorien von Seiendem und auch nach allen raumzeitlichen Modalitäten“46; „[j]edes Objekt, jeder Gegenstand überhaupt [...] bezeichnet eine Regelstruktur des transzendentalen Ego “47. Daraus folgt der regressive Charakter der transzendentalen Konstitution: Jede Art Konstitution muss eine besondere regionale Gattung als transzendentalen Leitfaden annehmen und die Identität eines Gegenstands dieser Gattung festhaltend, das noetisch-noematische Verhältnis zwischen der intentionalen Einheit des gemeinten Gegenstands und der Mannigfaltigkeit der subjektiven Gegebenheitsweisen, Meinungen und hyletischen Daten erhellen. ${ }^{48}$

44 Husserl E., Cartesianische Meditationen, $\$ 41$, hrsg. v. S. Strasser, Den Haag, M. Nijhoff, 1973, Hua I, S. 86-87.

45 Husserl E., Die Krisis der europäischen Wissenschaften und die transzendentale Phänomenologie, op. cit., $\$ 37$, Hua VI, S. 145.

46 Ibid., $\$ 48$, Hua VI, S. 169.

47 Husserl E., Cartesianische Meditationen, op. cit., \$22, Hua I, S. 90.

48 Ibid., \$21, Hua I, S. 88. 
Zweitens verliert sich Weltkonstitution keineswegs in eine offene, unbestimmte und chaotische Mannigfaltigkeit von einzelnen Konstitutionstypen; im Gegenteil wird die Ordnung der konstitutiven Typen vom Fundierungsverhältnis der regionalen Wesen geregelt, so dass die Folge der konstitutiven Analysen genau der Stufenfolge der materialen mundanen Gattungen entspricht: Weltkonstitution bestimmt sich also als die Gesamtaufgabe, ,in der Einheit einer systematischen und allumspannenden Ordnung am beweglichen Leitfaden eines stufenweise herauszuarbeitenden Systems aller Gegenstände möglichen Bewusstseins [...] alle phänomenologischen Untersuchungen als entsprechende konstitutive durchzuführen, also streng systematisch aufeinandergebaut, miteinanderverknüpf ${ }^{*}{ }^{49}$. Wenn der Lebensweltbegriff die Welt als den konkreten Horizont aller zugänglichen Gegenstände und Mitsubjekte und den Spielraum aller subjektiven Interessen besagt, muss aber methodisch diese konkrete Welt erstens durch das noematische Welteidos (d. h. durch die systematische Stufenfolge aller regionalen Gattungen von mundanen Objekten) ersetzt werden, und dieses zweitens durch das transzendentale Welteidos (d. h. durch die entsprechende Stufenfolge aller aufeinanderfundierten konstitutiven Typen).

Letztlich erscheint Lebenswelt im Rahmen der transzendentalen Phänomenologie keineswegs als Horizont einer Urgegebenheit, dessen Archifaktizität erkannt werden sollte. ${ }^{50}$ Im Gegenteil löst sie der transzendental methodische Prozess in eine systematische, geordnete und durchsichtige Folge von konstitutiven Stufen auf.

\section{Dingkonstitution und Weltkonstitution}

Ricœurs These entgegen gibt es also für Husserl „zwei mögliche[] Grundweisen, die Lebenswelt thematisch zu machen "51, die absolut nicht gleichwertig sind, sondern deren erste naiv bleibt, während die zweite allein absolut aufklärend erscheint. Einerseits gibt es die „naiv-natürliche Geradehineinstellung “ ${ }^{52}$, d. h. das in den Welthorizont Hineinleben oder das "ständig In-Weltgewißheit-leben“53, das direkte Gerichtetsein auf die Objekte und auf die vorgegebene Lebenswelt als

\footnotetext{
Ibid., $\$ 22$, Hua I, S. 90.

Cf. Tengelyi László, Welt und Unendlichkeit, Freiburg / München, Karl Alber Verlag, 2014, S. 180 ff. Husserl E., Die Krisis der europäischen Wissenschaften und die transzendentale Phänomenologie, op. cit., $\$ 38$, Hua VI, S. 146.

52 Ebenda.

53 Ibid., \$ 37, Hua VI, S. 145.
} 
den unendlichen Horizont für alle unsere Ziele und Zwecke. Andererseits gibt es dagegen die reflexiv orientierte „Einstellung auf das Wie der subjektiven Gegeben-

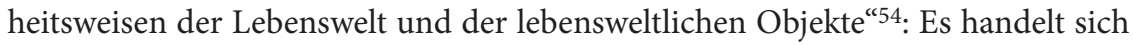
um eine konsequente und universale Umwandlung des thematischen Bewusstseins von der Welt, also um eine Wandlung der Geradehineinstellung und des direkten Dahinlebens in ein theoretisches Interesse für die „Objekte in ihrem Wie“.

Was besagt genau dieser letzte Ausdruck? Damit werden die Objekte im Wie ihrer subjektiven Gemeintheitsweisen und ihrer Gegebenheitsweisen aufgefasst: Damit werden erstens die Gegenstände insofern hingewiesen, als sie wahrnehmungsmäßig oder phantasiemäßig oder erinnerungsmäßig gemeint werden bzw. als sie mit noematischen Aktcharakteren affiziert werden; ${ }^{55}$ zweitens die Gegenstände insofern, als sie anschauungsmäßig wirklich gegeben werden und sich in sinnlichen einseitigen Aspekten abschatten, z. B. derselbe Würfel, der bald in Nah-, bald in Fernerscheinungen, bald von jener, von dieser Seite und in wandelnden Perspektiven erscheint, der sich mit jener oder dieser Gestalt oder Farbe oder mit irgendeiner sinnlichen Eigenschaft zeigt; ${ }^{56}$ drittens und korrelativ ergibt sich die ganze hyletische und noetische Seite, $d$. h. insgesamt die hyletischen oder sensuellen Daten, worauf alle sinnlichen Eigenschaften und Aspekte zurückweisen, und die subjektiven Meinungen, die auf solche Gegenstände gerichtet sind. In dieser Hinsicht kann Husserl vom theoretischen Interesse des Phänomenologen als einem "auf das Universum des Subjektiven ${ }^{\text {"57 }}$ gerichteten Interesse sprechen, wo unter "subjektiv“ doppeltes bedeutet wird: einerseits die noematischen Aktcharaktere und einseitigen Aspekte des Objekts, die als noematische offenbar nicht subjektiv im strengen und reellen Sinn sind; andererseits die Meinungen und Anschauungen, die dagegen streng subjektiv sind, da sie zur reellen Sphäre der subjektiven Erlebnisse und zum Gehalt des Bewusstseins gehören.

Jetzt möchten wir eine wichtige phänomenologische Frage stellen, die das Verhältnis zwischen Dingbewusstsein und Weltbewusstsein (bzw. Dingkonstitution und Weltkonstitution) angeht: Haben Ding- und Weltbewusstsein dieselbe Struktur, oder sind sie untereinander ganz verschieden? Ist im Fall der Welt transzendentale Konstitution wie im Fall des einzelnen Dinges möglich oder erweist sich die Welt als prinzipiell unkonstituierbar oder archifaktisch, also mit einem ursprünglichs-

\footnotetext{
Ibid., $\$ 38$, Hua VI, S. 146.

55 Husserl E., Ideen zu einer reinen Phänomenologie, Bd. I (= Ideen... I), \$131, hrsg. v. Karl Schuhmann, Den Haag, M. Nijhoff, 1976, Hua III/1, S. 303.

56 Husserl E., Cartesianische Meditationen, op. cit., $\$ 17$, Hua I, S. 78.

57 Husserl E., Die Krisis der europäischen Wissenschaften und die transzendentale Phänomenologie, op. cit., $\$ 38$, Hua VI, S. 149.
} 
ten Faktum identisch? Sind wir im Fall der Welt (d. h. des Seienden im Ganzen oder des Alls der Dinge) gezwungen, der transzendental-idealistischen These zu entsagen und die realistische These anzunehmen?

Eine wesentliche Heideggersche These annehmend hebt Husserl tatsächlich im $\$ 37$ der Krisis „einen grundsätzlichen Unterschied in der Weise des Weltbewusstseins und des Dingbewusstseins, des Objektbewusstseins " 58 , hervor: Einzelne Objekte sind nämlich bewusst „als Dinge, als Objekte im Welthorizont. Jedes ist ,etwas aus' der Welt" 59 ; das Einzelne ist „nichts für sich“, da dessen sinnliche Auffassung immer „Wahrnehmung in einem Wahrnehmungsfeld“ ist, während dieses Feld selbst „Ausschnitt, von der Welt“"60 ist. In Sein und Zeit erkennt Heidegger an, dass ein einzelnes Zeug nie „ist“, weil jedes sinnliche Seiende immer ein zuhandenes Zeug ist und jedes Zeug wiederum einer Zeugganzheit gehört, deren Struktur ein Komplex von praktischen Verweisungen ist und die letztlich zum Weltganzen und zum Dasein selbst zurückverweist. ${ }^{61}$ Ähnlich, aber abgesehen vom praktischen Charakter des Zuhandenen und sich nur in der Sphäre des sinnlich Wahrnehmungsmäßigen haltend, erkennt Husserl, dass jedes einzelne Objekt als „etwas aus der Welt" die Vorgegebenheit und Seinsgewissheit der Welt voraussetzt: Während das Objekt zum reinen Bewusstsein ganz und gar relativ bleibt und von ihm konstituiert wird, ist dagegen die Welt ein absolut Seiendes, dessen Sein jeder transzendentalen Konstitution vorangeht und das also Korrelat einer ursprünglichen Weltgewissheit ist; jedem endlichen Gegenstand gegenüber ist die Welt ein archifaktisches Seiendes, das sich jeder transzendentalen Konstitution und jeder Relativität zum Bewusstsein verweigert. ${ }^{62}$ Aber dies unter dieser bestimmten Voraussetzung: Das gilt nur in der naiven Geradehineinstellung des sich in der natürlichen Einstellung findenden Bewusstseins!

Im Gegenteil gibt es in der reflexiven Einstellung keinen Grundunterschied mehr zwischen Ding- und Weltkonstitution; beide Konstitutionsweisen haben nämlich dieselben wesentlichen Züge und Strukturen.

\footnotetext{
Ibid., $\$ 37$, Hua VI, S. 146.

Ebenda.

Ibid., $\$ 47$, Hua VI, S. 165.

Heidegger Martin, Sein und Zeit, \$15, Tübingen, Niemeyer, 1986, S. 68-71.

62 Husserl E., Erfahrung und Urteil, $\$ 7$, Hamburg, Glaassen \& Goverts, 1954 (= F. Meiner, 1999), S. 23-26. So spricht auch M. Merlau-Ponty in Le visible et l'invisible von der Welterfahrung als der „Vor-habe [pré-possession] einer Totalität, die da ist, noch bevor man weiß, wie und warum“ (Das Sichtbare und das Unsichtbare, übersetzt von Regula Giuliani und Bernhard Waldenfels, München, Fink, 3. Auflage, 2004 (= Übergänge. Bd. 13), S. 65 („pré-possession d’une totalité qui est là avant qu'on sache comment ni pourquoi“, Paris, Gallimard, 1965, S. 65).
} 
Erstens werden beide von einer ähnlichen synthetischen Struktur bestimmt: Ganz fern von der schon erwähnten These der transzendentalen Unkonstituierbarkeit der Welt liegt tatsächlich in der gesamten Aufgabe der transzendentalen Phänomenologie zu „entdecken, dass und wie Welt als Korrelat einer erforschbaren Universalität synthetisch verbundener Leistungen ihren Seinssinn und ihre Seinsgeltung in der Totalität ihrer ontischen Struktur gewinnt "63. Als Urform des Bewusstseins ist zugleich Synthesis die ursprüngliche Form jedes Objektbewusstseins und des Weltbewusstseins; ${ }^{64}$ ferner haben Dingbewusstsein und Weltbewusstsein dieselbe Horizontstruktur, so dass es einen strengen strukturellen Parallelismus zwischen dem Innenhorizont und dem Außenhorizont gibt. ${ }^{65}$ Für die Konstitution des einzelnen materiellen Dinges gilt nämlich die strukturelle Entgegensetzung und Zusammengehörigkeit von der Selbigkeit des Dinges und der Mannigfaltigkeit von dessen sinnlichen Darstellungen oder Gegebenheitsweisen; zum Beispiel stellt sich dasselbe Ding durch Wahrnehmungen des Sehdings und des Tastdings dar, das Sehding wiederum im Wandel der Perspektiven, der Nah- und Fernerscheinungen, und dessen Farbe wiederum durch Farbenabschattungen. ${ }^{66}$ Und die vollkommene oder adäquate Darstellung des einzelnen Dinges, d. h. der allseitigen Aspekte des Dinges, ist eine Kantische Idee, also eine unendliche Idee, die als geschlossene Ganzheit unzugänglich ist und einen unendlichen Prozess von sinnlichen Erscheinungen umfasst und vorzeichnet. ${ }^{67}$

Und genau dieselbe Struktur der „Darstellung-von“ gilt für Weltkonstitution! Es gibt nämlich einen genauen Parallelismus zwischen dem Innenhorizont des Dingbewusstseins und dessen Außenhorizont: Sowie sich das einzelne Ding „durch einen Kern ,originaler Präsenz “68 darstellt, d. h. durch den Kern seiner wirklich anschaulich gegebenen Aspekte, so stellt sich auch die Erfahrungswelt durch einen solchen Kern originaler Präsenz dar, nämlich den Kern des anschaulich gegebenen Wahrnehmungsfelds, und dieser wiederum durch den Kern des zentral wahrgenommenen Dings, um das herum sich ein Hof mitwahrgenomme-

63 Husserl E., Die Krisis der europäischen Wissenschaften und die transzendentale Phänomenologie, op. cit., $\$ 38$, Hua VI, S. 148.

64 Husserl E., Cartesianische Meditationen, op. cit., \$17, Hua I, S. 77.

65 Husserl E., Erfahrung und Urteil, op. cit., § 8, S. 27-29.

66 Husserl E., Die Krisis der europäischen Wissenschaften und die transzendentale Phänomenologie, op. cit., $\$ 45$, Hua VI, S. 161; Cart. Medit., $\$ 17$, Hua I, S. 78.

67 Husserl E., Ideen zu einer reinen Phänomenologie, op. cit., $\$ 143$, Hua III/1, S. 330-331; Cart. Medit., $\$ \$ 19,29$ u. 41, Hua I, S. 81-83, 98 u. 121.

68 Husserl E., Die Krisis der europäischen Wissenschaften und die transzendentale Phänomenologie, op. cit., $\$ 47$, Hua VI, S. 165. 
ner Daten bildet; ${ }^{69}$ in beiden Fällen von Ding- und Weltbewusstsein finden wir also dieselbe synthetische Struktur und dieselbe Korrelation von anschaulichem Kern und mitgemeintem Feld. ${ }^{70}$

Zweitens finden wir in beiden Fällen dieselbe idealistische Deutung der trans-

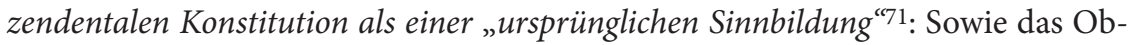
jekt einen Seinssinn hat (materielles Ding, Gebrauchsding, Lebewesen, usw.), der von einer Sinngebung des Bewusstseins gestiftet wurde, ${ }^{72}$ hat die Welt selbst einen Seinssinn, der auch von einer Sinnbildung oder Sinngebung gestiftet wurde ${ }^{73}$ - was in erster Linie von den geschichtlichen und kulturellen Bestimmungsprädikaten gilt, die ganz auffällig Produkte einer intentionalen Sinnbildung sind. Und sowie ferner das Objekt eine Seinsgeltung besitzt (Wirklichsein, Möglichsein, Notwendigsein, Zweifelhaftsein usw.), die sich in den subjektiven Evidenzen des Bewusstseins ausweisen kann, so hat auch die Welt als Ganzes eine Seinsgeltung.

Aber in diesem letzten Fall müssen wir zwei Weltbegriffe unterscheiden: Da einerseits Leben „ständig In-Weltgewissheit-leben“74 ist, hat und bewährt die Welt als horizonthafter Boden aller Erfahrung immer, ständig und notwendig dieselbe Seinsgeltung, nämlich die des in Seinsgewissheit Vorgegebenen und daher notwendig Seienden. Sobald wir aber die phänomenologische Einstellung einnehmen, erreichen wir eine neue Einstellung „über der Geltungsvorgegebenheit der Welt" ${ }^{\text {"75 }}$, so dass das Denken „über sein natürliches Sein [scil. des Seienden] und über die natürliche Welt gestell" "76 wird. Da in der von dieser neuen Einstellung erschlossenen Sphäre eine strenge ontologische Korrelation zwischen Seiendem jeder Art und transzendentaler konstituierender Subjektivität gilt, ${ }^{77}$ hat darin Seiendes Seinssinn und Seinsgeltung, die nur Korrelate von gewissen subjektiven sinngebenden Meinungen und seinausweisenden Evidenzen sind, und die also dem Bewusstsein nicht vorgegeben sind; die Welt als so und so konkret seiende, als alle konkreten Dinge umfassender Horizont, wird charakterisiert durch die Seinsgeltung des Zu-

69 Husserl E., Die Krisis der europäischen Wissenschaften und die transzendentale Phänomenologie, op. cit., $\$ 40$, Hua VI, S. 152.

70 Ibid., $\$ 47$, Hua VI, S. 165.

71 Ibid., $\$ 49$, Hua VI, S. 170.

72 Husserl E., Ideen zu einer reinen Phänomenologie, op. cit., \$55, Hua III/1, S. 120.

73 So beschreibt Husserl Phänomenologie als eine „Wissenschaft von dem universalen Wie der Vorgegebenheit der Welt“, die ihre Kraft „aus ihrer letzten Sinngebung schöpft“ (Husserl E., Die Krisis der europäischen Wissenschaften und die transzendentale Phänomenologie, op. cit., \$38, Hua VI, S. 149).

74 Ibid., $\$ 37$, Hua VI, S. 145.

75 Ibid., $\$ 40$, Hua VI, S. 153.

76 Ibid., $\$ 41$, Hua VI, S. 155.

77 Ibid., $\$ 41$, Hua VI, S. 154. 
fälligen und Zweifelbaren und gewinnt eine positive Seinsgeltung erst durch die rechtfertigenden Evidenzen des Bewusstseins.

Zum Schluss können wir also sagen, dass die Entgegensetzung von Ding- und Weltbewusstsein nur in der natürlichen Einstellung gilt, die mit ontologischer Naivität behaftet ist und in deren Naivität die vorgegebene Welt beschrieben werden kann; aber sobald als wir die transzendentale Einstellung einnehmen, verschwindet für die Welt dieser natürliche Status ontologischer Ausnahme: Dann ist sie wie jedes endliche und einzelne Seiende nur ein Korrelat der subjektiven Meinungen und Evidenzen des Bewusstseins, dessen Sinn und Geltung sich fortschreitend bewähren und bestimmen. Als das All des Seienden ist die Welt in der transzendentalen Sphäre keine Ausnahme innerhalb des Seienden.

\section{Ein Grundproblem der transzendentalen Weltkonstitution: das Problem des Fundierungsverhältnisses und der aufgeschichteten Konstitution}

Vergleichen wir endlich noch einmal die Konstitution des einzelnen Objekts und die der allumfassenden Welt, um eine spezifische methodische Schwierigkeit der transzendentalen Konstitution herauszustellen.

Heben wir zuerst die Struktur der transzendentalen Konstitution des einzelnen Gegenstands hervor. Alle konstitutiven Arbeitsprobleme ordnen sich unter den Titeln ego - cogito - cogitatum oder ego - cogitatio - cogitata, ${ }^{78}$ wobei das Ego der leistende und konstituierende Ichpol bezeichnet, bzw. das cogitatum den korrelativen intentionalen Gegenstandspol in seiner sichdarstellenden Selbigkeit bezeichnet, und die cogitatio ein allgemeiner Titel für die verschiedenen subjektiven Weisen der intentionalen Richtung auf etwas Gegenständliches ist. In dieser dreifachen Struktur besitzt der gegenständliche Pol eine wesentliche Funktion: Nämlich diejenige eines intentionalen Indizes oder eines transzendentalen „Leitfadens für die Rückfrage nach den Mannigfaltigkeiten der Erscheinungsweisen und ihren intentionalen Strukturen“79 - und dies der allgemeinen Thesis nach, wonach jeder Gegenstand oder jeder Gegenstandstypus dem transzendentalen Ego eine Regelstruktur vorzeichnet: Jeder Konstitutionstypus hat seinen eigenen und bestimmten strukturellen Stil der Bewährungsweise, der vom Eidos oder von der materialen Gattung des Gegenstands vorgezeichnet wird. ${ }^{80}$ Das konstitutive

\footnotetext{
Ibid., $\$ 50$, Hua VI, S. 173-175.

Ibid., $\$ 50$, Hua VI, S. 175.

80 Husserl E., Cartesianische Meditationen, op. cit., $\$ \$$ 20, 21, 22 u. 29, Hua I, S. 85, 88, 90 u. 98-99: „Jedes Objekt, jeder Gegenstand überhaupt [...] bezeichnet eine Regelstruktur des transzendentalen
} 
Apriori wird also nur vom materialen oder gegenständlichen Apriori bestimmt und nicht von der spezifischen (endlichen oder unendlichen, rezeptiven oder schöpferischen) Natur und Struktur des transzendentalen Subjekts (d. h. der Mannigfaltigkeit seiner Vermögen).

Gilt aber dieses Prinzip auch für die Weltkonstitution? Oder hat diese Leitfadenfunktion für die Welt als das All der Dinge keinen bestimmten Sinn, da der formale allumfassende Horizont aller Erfahrungen keinen konkreten Sinngehalt hätte?

Unserer Meinung nach gilt dieses Prinzip auch für Weltkonstitution, aber $m u$ tatis mutandis. Es muss nämlich eine Ontologie der Lebenswelt eine „feste Typik“ herausstellen, die eine „methodisch als reines Apriori zu umgreifende Wesenstypik ist" ${ }^{\text {" }}$. Worin besteht eine solche Wesenstypik? Sie umfasst alle wesentlichen Allheitsstrukturen oder allgemeinen Strukturformen, die zu einer Erfahrungswelt überhaupt gehören (Zeit, Raum, Materie, Leben, usw.); 82 und jede dieser Strukturformen entspricht zugleich einem besonderen regionalen Erfahrungsgebiet von einzelnen Objekten (res temporalis, res extensa, res materialis, Lebewesen, usw.) ${ }^{83}$. Wie soll die Aufgabe der transzendentalen Konstitution diesen Strukturformen und Gebieten gegenüber methodisch verfahren? Es ist einfach, dieser Frage eine Antwort zu geben: Innerhalb der phänomenologischen Epoché hat der Phänomenologe immer die theoretische Freiheit, die thematische Blickrichtung bald auf diese, bald auf jene intentionale Schicht zu orientieren und zu konzentrieren, und eine besondere gegenständliche Gattung oder eine besondere Schicht dieser Gattung als transzendentalen Leitfaden zu nehmen, um die phänomenologische Reflexion zu üben und die zugehörigen Mannigfaltigkeiten von Erscheinungsweisen aufzuklären; die transzendentale Konstitution des Gegenstands überhaupt kann

Ego.“ (S. 90). Ideen... I, \$ 149, Hua III/1, S. 344: „Jede gegenständliche Region konstituiert sich bewusstseinsmässig. Ein durch die regionale Gattung bestimmter Gegenstand hat als solcher, sofern er wirklich ist, seine a priori vorgezeichneten Weisen, wahrnehmbar, überhaupt klar oder dunkel vorstellbar, denkbar, ausweisbar zu sein." Ideen... III, $\$ \$ 3$ u. 7, hrsg. v. M. Biemel, Den Haag, M. Nijhoff, 1971, Hua V, S. 13, 34 u. 36: „Das gilt für alle Seinskategorien, die korrelativ auf kategoriale Grundformen gebenden Bewusstseins zurückführen." (S. 13). Diese Grundthese des Husserlschen Denkens haben wir in unserem Buch Par-delà la révolution copernicienne [Jenseits der Copernikanischen Wendung] (Paris, Presses Universitaires de France, 2012) zum methodischen Leitfaden einer Gesamtdeutung der Husserlschen trasnzendentalen Philosophie gemacht. Husserl E., Die Krisis der europäischen Wissenschaften und die transzendentale Phänomenologie, op. cit. $\$ 51$, Hua VI, S. 176. Hua IX, S. 67. 
also im Wandel der partiellen thematischen oder intentionalen Orientierungen aufgeklärt werden. ${ }^{84}$

Aber welcher methodischen Ordnung soll nun die Gesamtkonstitution der Welt als des Alls des Seienden folgen? Ist es möglich, von einer solchen Gesamtkonstitution der Welt selbst zu sprechen, oder sind wir im Gegenteil dazu gezwungen, den Sinn der transzendentalen Konstitution auf einzelne Objekte und Gegenstandstypen zu beschränken?

Das Wesentlichste und Entscheidendste ist hier, der „wesensmäßigen Fundierungsordnung [der gegenständlichen Schichten oder Stufen zu] folgen “85. Was bedeutet diese methodische Ausweisung? Sie besagt, dass die erdenklichen Gegenstandstypen kein Chaos bilden, sondern umgekehrt ein streng geordnetes System von aufeinander fundierten gegenständlichen Wesen, wobei das Fundierungsverhältnis ein Doppeltes bedeutet: erstens, dass die höhere Stufe die niedrige voraussetzt, und zweitens, dass die höhere Stufe sich auf die niedrige aufbaut; ${ }^{86}$ so setzt jedes ausgedehnte Objekt ein Zeitobjekt insofern voraus, als es eine zeitliche Dauer notwendig erfüllt, und baut sich zugleich der Sinn eines jeden ausgedehnten Objekts auf der vorgegebenen Schicht des Zeitobjekts auf. ${ }^{87}$ Daraus ergibt sich für den Inbegriff der gegenständlichen Wesen eine Ordnungsbeziehung, wonach deren ganze Stufenfolge sich auf der niedrigsten Unterlage des Zeitobjekts (res temporalis) aufbaut: res temporalis, res extensa, res materialis, Lebewesen, Animalien, Personen, Wert- oder Bestimmungsprädikate, soziale Gemeinschaften, materiale und formale Idealitäten usw. - wobei nicht entschieden ist, ob die Stufenfolge geschlossen oder nicht ist. Vom faktischen Weltbegriff als dem des Alls der Seienden oder des universalen Horizonts aller Erfahrung müssen wir also einen eidetischen Weltbegriff unterscheiden: Das Eidos „Welt" befasst alle wesentlichen Schichten der Gegenständlichkeit und das Fundierungsverhältnis, wonach diese Stufen in einer universalen Ordnung von unten auf gesetzt werden. ${ }^{88}$

Welche methodische Notwendigkeit folgt daraus für die transzendentale Konstitution? Da überall das Wesen des Gegenstands den transzendentalen Leitfaden für die Konstitution bildet, wird die gesamte Fundierungsordnung der Wesen

84 Husserl E., Die Krisis der europäischen Wissenschaften und die transzendentale Phänomenologie, op. cit., $\$ 51$, Hua VI, S. 177.

85 Ibid., \$34a, Hua VI, S. 127.

86 Husserl E., Vorlesungen über Ethik und Wertlehre, C, $\$ 3$, hrsg. v. U. Panzer, Dordrecht, Kluwer Academic Publishers, 1996, Hua XXVIII, S. 252, Fussnote: „Fundiert im doppelten Sinne: 1) sich auf etwas bauen; 2 ) es als notwendig voraussetzen."

87 Husserl E., Ideen zu einer reinen Phänomenologie, op. cit., \$ 149, Hua III/1, S. 347-348.

88 Diese Unterscheidung haben wir nicht ausdrücklich in Husserls Werken gelesen, aber dennoch lässt sie sich aus der Dritten Untersuchung und aus den letzten Paragraphen der Ideen... I herleiten. 
den transzendentalen Gesamtleitfaden für Weltkonstitution bilden; demnach müssen sich alle konstitutiven Typen notwendig ,in [die] Einheit einer systematischen und allumspannenden Ordnung "89 einordnen. Die transzendentale Weltkonstitution soll methodisch von unten auf und stufenweise aufgeklärt werden, mit der niedrigsten Unterlage des Zeitobjekts beginnend, dem Fundierungsverhältnis der eidetischen Schichten folgend und die transzendentale Reflexion auf jeder gegenständliche Stufe vollziehend, um die transzendentale Konstitution des jeweiligen Gegenstandstypus aufzuhellen.

Über diesen methodischen, progressiven und universalen Charakter der transzendentalen Weltkonstitution als einer sich von unten auf aufbauenden und stufenweise fortschreitenden Konstitution drücken sich zwei Texte besonders klar aus. Erstens können wir im \$22 der Cartesianischen Meditationen lesen:

Es deutet sich eine ungeheure Aufgabe an, welche die der gesamten transzendentalen Phänomenologie ist, die Aufgabe, in der Einheit einer systematischen und allumspannenden Ordnung am beweglichen Leitfaden eines stufenweise herauszuarbeitenden Systems aller Gegenstände möglichen Bewusstseins [...] alle phänomenologischen Untersuchungen als entsprechende konstitutive durchzuführen, also streng systematisch aufeinander gebaut, miteinander verknüpft. ${ }^{90}$

Und zweitens finden wir in der Vorlesung von 1919 über Natur und Geist das folgende Zitat, das wie ein Husserlscher Discours de la méthode lautet und den progressiven methodischen Charakter der Konstitution in reiner Klarheit ausdrückt:

Die Welt hat ihren apriorischen Stufenbau, und es ist offenbar notwendig, diesem Aufbau auf ontischer Seite in der phänomenologisch konstitutiven Untersuchung zu folgen, also mit der untersten Stufe zu beginnen und dann den sich aufstufenden Schichten nachzugehen. Selbstverständlich ist ja das ursprünglich gebende Bewusstsein einer fundierten Gegenständlichkeit komplizierter als das der fundierenden. [...] Die Unterstufe ist aber unter allen Umständen die der physischen Natur. ${ }^{91}$

Während in den Logischen Untersuchungen die wesentlichste Forderung der deskriptiven Psychologie die der reinen Beschreibung war, drückt sich hier umgekehrt ganz deutlich das Primat der Wesensanschauung und der Cartesianischen Methode gegenüber dem Imperativ der reinen Beschreibung aus; allerdings for-

89 Husserl E., Cartesianische Meditationen, op. cit., $\$ 22$, Hua I, S. 90.

90 Ebenda - wir unterstreichen.

91 Husserl E., Natur und Geist (Vorlesungen Sommersemester 1919), hrsg. v. M. Weiler, Hua Materialien Bd. IV, S. 150. 
muliert Husserl in Erfahrung und Urteil eine methodische Forderung, die genau wie die dritte Regel der Cartesianischen Methode klingt: „Es ist ja methodisches Gebot, bei solchen Analysen mit dem Einfachen zu beginnen und dann erst zum Komplizierten aufzusteigen "92. Letztlich können wir also sagen, dass Husserls konstitutive Analysen der zweiten und der dritten Regel der Cartesianischen Methode folgen: Zuerst dem Prinzip der methodischen Zergliederung jeder theoretischen Schwierigkeit in einfache Teile, wonach die universale Aufgabe der transzendentalen Konstitution den verschiedenen eidetischen Schichten der Gegenständlichkeit nach zergliedert wird; und dann dem Prinzip des methodischen In-Ordnung-Setzens der konstitutiven Analysen, wonach sie auf der noetischen Seite dem Fundierungsverhältnis der eidetischen noematischen Stufen folgen.

Prima facie erscheinen diese methodischen Betrachtungen ganz selbstverständlich: ist es nicht ein einfaches Gebot guter und gesunder Methode, mit dem Einfachen zu beginnen und erst nachher mit dem Komplizierten fortzuschreiten?

$\mathrm{Ob}$ darin aber nicht trotzdem etwas vom phänomenologischen Standpunkt aus gesehen Problematisches liegt, können wir aus der Folge des Cartesianischen Zitats erahnen: „et supposant même de l'ordre entre ceux qui ne se précèdent point naturellement les uns les autres ${ }^{\text {"993 }}$ - und gar Ordnung unter denen voraussetzend, wobei keine den anderen natürlich vorangehen: D. h. die Ordnung zwischen den Teilen eines Problems kann auch der Natur der Sache selbst ganz äußerlich und ausschließlich Produkt des methodischen Verfahrens sein.

Dass wird sich außerdem aus dem Unterschied und der Gegenübersetzung von naturalistischer und personalistischer Einstellung im zweiten Band der Ideen bestätigen.

Nämlich wird von Husserl in den Ideen... II ausdrücklich ausgesagt, dass die transzendentale Konstitution der naturalistischen Welt geschichtet ist und dass sie der Fundierungsordnung der eidetischen Schichten der Welt folgt. In der naturalistischen Einstellung, die ausschließlich an der sinnlichen Natur orientiert bleibt und jedes kulturelle Bedeutungsprädikat ausschließt, sind nämlich „die konstituierenden Grundarten der Auffassung [...] aufeinander gebaut"94, so dass die Konstitution sich stufenweise und von unten auf aufklären lässt: Man beginnt mit

92 Husserl E., Erfahrung und Urteil, op. cit., § 14, S. 69. Vgl. Descartes Rene, Discours de la méthode, II: „Le troisième [précepte était], de conduire par ordre mes pensées, en commençant par les objets les plus simples et les plus aisés à connaître, pour monter peu à peu comme par degrés jusques à la connaissance des plus composés" (Adam-Tannery, VI, S. 18, CEuvres complètes, Bd. III (hrsg. v. J.-M. Beyssade u. D. Kambouchner), Paris, Gallimard, 2009, S. 93).

93 Descartes R., Discours de la méthode, op. cit., II (AT VI, S. 18, Euvres complètes, III, S. 93).

94 Husserl E., Ideen... II: Phänomenologische Untersuchungen zur Konstitution, $\$ 49 a$, hrsg. v. M. Biemel, M. Nijhoff, 1954, Hua IV, S. 174. 
der Grundschicht der wahrnehmungsmäßigen Erfahrung des materiellen Dinges, fortschreitend konstituiert man den fremden Leib als Unterlage der ästhesiologischen Schicht, daraufhin konstituiert man den fremden Menschen durch Einfühlung, dann die Gebrauchsobjekte durch Urstiftung der Bedeutungsprädikate usw. Es handelt sich um eine progressive Konstitution, die von unten auf, d. h. von der Unterlage des materiellen Dinges aus bis zu den höheren Stufen aufwärts verfährt.

Im Gegensatz dazu finden wir bei der personalistischen alltäglichen und ganz natürlichen (nicht naturalistischen) Einstellung keine progressiv und stufenweise strukturierte Auffassung, also keine geschichtete und sich dem Fundierungsverhältnis nach stufenweise aufbauenden Auffassung. Zum Beispiel setzt die Erfahrung der fremden Person keine „Introjektion der Seele ${ }^{\text {“95 }}$ voraus, die zuerst den fremden Leib als bloßes materielles Ding wahrnehmen müsste, um erst nachher auf dessen Unterlage den Oberbau des psychischen Lebens hineinzusetzen; sondern umgekehrt ist sie unmittelbar „komprehensive Erfahrung vom Dasein des Anderen“96, durch welche wir das fremde Subjekt „ohne weiteres als personales Subjekt und dabei auf Objektitäten bezogen, auf die auch wir bezogen sind“97, auffassen; „In dieser Einstellung fällt es [uns] gar nicht ein, den Geist dem Leibe ,einzulegen, d. i. als etwas am Leib, als in ihm Fundiertes, mit dem Leibe zu einer Realität Gehöriges zu betrachten"98. Der wesentliche Punkt ist dabei, dass wir in der personalistischen Einstellung keine fundierungsmäßige Erfahrung oder Auffassung des anderen Subjekts haben: Den Anderen fasse ich keineswegs von unten auf als einen materiellen Gegenstand, der übrigens mit seelischen und geistigen Bestimmungen begabt wäre, sondern direkt auf der Stufe des geistigen und sozialen Sinnes. Dank der Akte der sozialen Wechselbeziehung oder der spezifisch sozialen und kommunikativen Akte sind uns die Anderen nicht als Gegenstände anschaulich gegeben, sondern als „Genossen“ oder als „Gegensubjekte" 99 , d. h. als Glieder eines kommunikativen Verbands und eventuell einer sozialen Subjektivität höherer Ordnung (Universität, Stadt, Region, Staat, Menschentum, religiöse Gemeinschaft usw.). In dieser sozialen Erfahrung sind wir unmittelbar auf die Welt der sozialen Intersubjektivität und der korrelativen sozialen und kulturellen Objektivitäten bezogen und finden uns direkt auf der Stufe der geistigen Welt als des Gesamtinbegriffs dieser subjektiven Gemeinschaften und Objektivitäten. ${ }^{100}$

\footnotetext{
Ibid., $\$ 49 \mathrm{a}, \mathrm{Hua}$ IV, S. 175.

Ibid., $\$ 51$, Hua IV, S. 191.

Ebenda.

Ibid., $\$ 51$, Hua IV, S. 190.

Ibid., \$ 51, Hua IV, S. 194.

100 Ibid., § 51, Hua IV, S. 196-197.
} 
Und dasselbe gilt ähnlich für die Umwelt der Gebrauchsobjekte oder der $\pi \rho \dot{\gamma} \gamma \mu a \tau \alpha$ : Diese Sphäre der umweltlichen Dinge ist „als Wirklichkeit für den Geist immerzu auch begeistete, geistig bedeutsame, geistigen Sinn in sich tragend"101. Zum Beispiel sehe ich unmittelbar Kohle als Heizmaterial, also als dienlich und zum Heizen dienend, und nicht zuerst als bloßes materiales Ding, das darüber hinaus nach der sinnlichen Wahrnehmung bewertet werden und aus diesem Bewertungsakt neue Wertprädikate in sich aufnehmen könnte. In der personalistischen, praktischen und kulturellen Blickrichtung zur Umwelt und zu den Umweltdingen wird das Ding unmittelbar auf der Stufe der kulturellen Bestimmungsprädikate als Gebrauchsding aufgefasst; dessen Seinssinn wird also nicht von einer bewertenden und deutenden Aktivität als eine neue Seinsdimension oder als eine neue Prädikatenstufe auf der Unterlage der materiellen Natur aufgebaut, sondern direkt vom Verständnis der praktischen und kulturellen Sinnesstufe aus aufgefasst.

Letztlich zeigt sich eine Umwendung der perspektivischen Ordnung, wenn wir von der naturalistischen bis zur personalistischen Einstellung fortschreiten: Am methodischen Cartesianischen Gebot des Fortgangs vom Einfacheren bis zum Komplizierteren orientiert verfährt die transzendentale Konstitution von unten auf, d. i. stufenweise von der niedrigsten Stufe der materiellen Natur bis zu den höheren Stufen der intersubjektiven Erfahrung und der Kultur; umgekehrt hat immer die umweltliche Auffassung eines jeden Gegenstands von der höheren Stufe des intersubjektiven und kulturellen Sinnes aus Priorität, d. h. von oben an, so dass auch die bloße Natur mit Bestimmungsprädikaten begabt ist. Es gibt also eine strenge Unverträglichkeit zwischen den methodischen Cartesianischen Forderungen der transzendentalen Konstitution und der rein deskriptiven Aufgabe einer Phänomenologie des reinen Bewusstseins. Die Aufgabe der transzendentalen Konstitution ist grundsätzlich eine genetische Aufgabe, deren eigenes Ziel bleibt, den (nicht psychologischen) Ursprung des Seienden selbst (und nicht der bloßen Vorstellung des Seienden) von den noetischen Aktivitäten und Evidenzweisen des reinen Bewusstseins aus klarzumachen; eine solche Aufklärung des subjektiven Sinnesurprungs des Seienden muss notwendig stufenweise und progressiv verfahren, um den Aufbau dieses Sinnes ans Licht zu bringen. Umgekehrt ist die Aufgabe einer Phänomenologie des Bewusstseins eine rein deskriptive, und keine genetische: um das Bewusstsein der alltäglichen Umwelt oder eines Umweltdinges aufzuklären, muss man sich lediglich in diese Bewusstseinsweise hineinversetzen, um die phänomenologische Reflexion zu vollziehen und die Gegebenheitsweise der Umwelt oder des Dinges ans Licht zu bringen; dann verschwinden die Car-

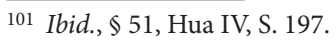


tesianischen Regeln der Zergliederung, des Inordnungsetzens und der progressiven Konstruktion, die nunmehr durch den theoretischen Imperativ der reinen Beschreibung und der Aufmerksamkeit auf die Sinnverweisungen der Umwelt ersetzt werden.

\section{Schlusswort}

Zum Schluss ist es nach diesen Analysen der Lebenswelt möglich geworden, einige Ergebnisse herauszuheben.

Erstens darf man nicht, wie Ricoeur es tut, die Lebenswelt als eine absolut letzte und unabhängige Vorgegebenheit verabsolutisieren: Nur hinsichtlich der wissenschaftlichen idealisierten Welt hat sie eine gründende (aber relative) Funktion;

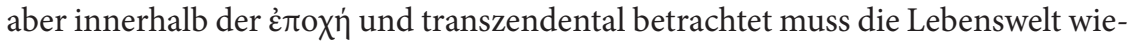
derum konstituiert werden.

Zweitens haben wir eine Spannung und vielleicht einen Widerspruch ans Licht gebracht: nämlich den Widerspruch zwischen der Erscheinungs- oder Auffassungsweise der Lebenswelt in der personalistischen Einstellung und der Konstitution der Lebenswelt in der reflexiv-transzendentalen Einstellung; durch die alltägliche Auffassung wird die Lebenswelt immer schon als begeistete und von oben an gegeben, während deren transzendentale Konstitution immer stufenweise, von unten auf und progressiv thematisiert und aufgeklärt wird. Die Forderungen der Cartesianischen Methode, d. h. des methodischen Beginnens mit dem Einfachsten und des progressiven Fortgangs vom Einfachen zum Komplizierten, sind also den wesentlichen und innerlichen Strukturen der Phänomenalität entgegengesetzt.

Drittens und letztens steht es zu vermuten, dass die Lebenswelt als Begriff der philosophischen Anthropologie zur Ontologie der alltäglichen Erfahrungswelt und nicht zur transzendentalen Konstitution gehört. Wie Alfred Schütz schon im Jahre 1959 schrieb: „Man kann vermuten, dass Intersubjektivität kein konstitutives Problem sei, das innerhalb der transzendentalen Sphäre zu lösen sei, sondern eine Gegebenheit der Lebenswelt. Sie ist eine ontologische Grundkategorie des menschlichen In-der-Welt-Sein und damit von jeder philosophischen Anthropologie"102.

102 Schutz Alfred, „Le problème de l'intersubjectivité transcendantale chez Husserl“ [„Das Problem der transzendentalen Intersubjektivität bei Husserl“], in Husserl, Cahiers de Royaumont, Philosophie $\mathrm{n}^{\circ}$ III, Paris, Minuit, 1959, S. 362-363: „On peut supposer que le problème de l'intersubjectivité n'est pas un problème de constitution à résoudre à l'intérieur de la sphère transcendantale, mais une donnée du monde de la vie. Elle est la catégorie ontologique fondamentale de lêtre de l'homme dans le monde et par là même de toute anthropologie philosophique“. 
Es gab nämlich im Husserlschen Denken hinsichtlich des Konstitutionsbegriffs eine wichtige Sinnverschiebung: Während die Konstitution in den Logischen Untersuchungen lediglich eine intentionale Auslegung des Sinnes der Akte bedeutete, wurde sie nachher von Husserl idealistisch als Aufklärung der Art und Weise gedeutet, wie der Sinn und das Sein des Seienden von den intentionalen Meinungen des reinen Bewusstseins erzeugt und von dessen ursprünglich gebenden Evidenzen gerechtfertigt werden. Sicherlich ist es möglich und notwendig, die intentionalen und sozialen Akte auszulegen, welche die Sinnesdimensionen der sozialen Umwelt meinen - dies aber nur innerhalb einer Phänomenologie der natürlichen Einstellung; vielleicht erweist es sich aber als unmöglich, die transzendentale und genetische Erzeugung der Sinnschichten der Welt ohne schwierige Voraussetzungen aufzuklären.

Dominique Pradelle ist Professor für zeitgenössische Philosophie an der Université Paris Sorbonne-Paris IV (seit 2013) und Direktor des Husserl-Archivs in Paris. Er ist spezialisiert auf das Studium der deutschen Philosophie der Gegenwart, der Metaphysik und der Phänomenologie sowie der Philosophie der Mathematik. Autor der Bücher: Par-delà la révolution copernicienne - Sujet transcendental et facultés chez Kant et Husserl und Généalogie de la raison - Essai sur l'historicité du sujet transcendental de Kant à Heidegger.

E-mail : Dominique.Pradelle@sorbonne-universite.fr 